Case Report

\title{
First Report of Ventriculoperitoneal Shunt Infection due to Cyberlindnera fabianii
}

\author{
Jonathan Baghdadi, ${ }^{1}$ Peera Hemarajata, ${ }^{2}$ Romney Humphries, ${ }^{2}$ and Theodoros Kelesidis ${ }^{1}$ \\ ${ }^{1}$ Division of Infectious Diseases, Department of Medicine, David Geffen School of Medicine at UCLA, Los Angeles, CA 90095, USA \\ ${ }^{2}$ Department of Pathology \& Laboratory Medicine, David Geffen School of Medicine at UCLA, Los Angeles, CA 90095, USA \\ Correspondence should be addressed to Theodoros Kelesidis; tkelesidis@mednet.ucla.edu
}

Received 17 July 2015; Accepted 19 October 2015

Academic Editor: Gernot Walder

Copyright (c) 2015 Jonathan Baghdadi et al. This is an open access article distributed under the Creative Commons Attribution License, which permits unrestricted use, distribution, and reproduction in any medium, provided the original work is properly cited.

Fungal infections in the central nervous system (CNS) are associated with significant morbidity and death. Transient fungemia in immunocompetent patients without any other risk factors for fungemia has been suggested as a possible mechanism that may lead to serious fungal ventriculoperitoneal (VP) shunt infections, but evidence is lacking. The clinical spectrum, diagnosis, and optimal therapy of Cyberlindnera fabianii infections remain to be determined. We describe the first case of CNS infection due to C. fabianii that occurred in an immunocompetent adult with a VP shunt. Spontaneous translocation with yeast that is not part of the normal gastrointestinal flora in the setting of ingestion of multiple servings of a fermentation product was the likely source from which Cyberlindnera fabianii gained entrance into the VP shunt system, causing meningitis in this patient. The authors conclude that, in view of the high morbidity associated with yeast infection of the CNS, long-term antifungal therapy should be strongly considered in cases where the VP shunt cannot be completely removed. Transient fungemia may lead to invasive disease in an immunocompetent host with VP shunt, even in the absence of any other risk factors for fungemia and even after remote placement of the VP shunt.

\section{Introduction}

Ventriculoperitoneal (VP) shunt is one of the most common neurosurgical procedures. Although infection is a common complication of shunt procedures, fungi are rarely implicated in VP shunt infections [1-4]. Transient fungemia and secondary colonization of VP shunts in the absence of other risk factors for fungemia have been suggested as a possible mechanism of fungal VP infections [5] but definite evidence is lacking. The heterothallic yeast Cyberlindnera fabianii, previously known as Hansenula fabianii, Pichia fabianii, and Lindnera fabianii [6], belongs to the phylum Ascomycota and is a member of the same order (Saccharomycetales) as other medically important yeasts such as Candida spp. Results from a recent phylogenetic analysis based on large and small subunit ribosomal ribonucleic acids (rRNA) and translation elongation factor- $1 \alpha$ gene led to separation of the genus Cyberlindnera from its parental genus Williopsis [7]. Only 14 infections due to this organism have been reported [8-16].
Of the 14 cases, 10 involved fungemia [9-16] and 4 were limited to the genitourinary tract $[8,16]$. Herein, we report the first case of central nervous system (CNS) infection due to $C$. fabianii that also suggests that transient fungemia in immunocompetent patients without any other risk factors for fungemia and with a remote history of VP placement may lead to serious fungal VP shunt infections.

\section{Case Report}

A 49-year-old Vietnamese-American woman with history of depression presented to our institution with confusion, headache, and difficulty in walking. Her medical history was significant for a ruptured anterior communicating artery aneurysm 10 years earlier that required craniotomy, clipping, and placement of a ventriculoperitoneal (VP) shunt. Four months prior to her presentation, she underwent a second craniotomy for clipping of a right middle cerebral artery aneurysm that was found on surveillance imaging. 
On presentation, she denied fever, chills, neck stiffness, photophobia, or head trauma. She was afebrile. The rest of her vital signs and physical examination, including neurologic examination, were unremarkable. Laboratory data was significant for a white blood cell count of 7810 cells $/ \mathrm{mm}^{3}$ with $66.6 \%$ polymorphonuclear cells. Other laboratory data were within normal limits. Imaging (computed tomography) of the neck, chest, and abdomen showed hydrocephalus and the VP shunt in position without kinks.

Shortly after presentation, the patient underwent urgent neurosurgery for VP shunt revision. Close examination of the shunt during surgery revealed an obstructed valve, which was replaced. The intraventricular portion of the shunt could not be removed for inspection due to tissue scarring. Postoperatively, the patient became somnolent and was intubated for airway protection. Cerebrospinal fluid (CSF) studies from samples taken intraoperatively were significant for 29 red blood cells per $\mathrm{mm}^{3}$, 11 white blood cells per $\mathrm{mm}^{3}$ (33\% neutrophils, 55\% lymphocytes, and $12 \%$ monocytes), protein $65 \mathrm{mg} / \mathrm{dL}$, and glucose $29 \mathrm{mg} / \mathrm{dL}$. CSF Gram stain demonstrated budding yeast. Other studies, including serum and CSF cryptococcal antigen (ImmunoMycologics, Inc., Norman, OK), Coccidioides IgM and IgG antibodies (Meridian Bioscience, Inc., Cincinnati, $\mathrm{OH}$ ), and bacterial and fungal blood cultures, were negative.

She was started empirically on intravenous liposomal amphotericin B at a dose of $5 \mathrm{mg} / \mathrm{kg}$ daily and oral flucytosine at a dose of $25 \mathrm{mg} / \mathrm{kg}$ four times daily on hospital day 1 . Her mental status improved on antifungal therapy and she was extubated on hospital day 3. Though fungal CSF culture from hospital day 3 showed growth of a yeast after 10 days of incubation, CSF specimens collected on hospital days 5 and 7 were negative for microorganisms, both by fungal and Gram stain, and culture. A second neurosurgical procedure was performed, but the deep intraventricular portion of the VP shunt still could not be removed due to severe tissue scarring. Instead, that end was ligated, the remainder of the shunt was removed, and a new shunt was placed on the contralateral side.

The yeast isolated from CSF collected during the first neurosurgical procedure demonstrated lush growth after 24 hours of incubation on inhibitory mold agar (IMA) [17] at $30^{\circ} \mathrm{C}$. Two colony morphotypes (smooth and rough off-white colonies) were observed. Wet mount preparation from both morphotypes demonstrated budding yeast cells with pseudohyphae (Figure 1). Both morphotypes were identified by a Vitek MS Matrix-Assisted Laser Desorption/Ionization Time of Flight (MALDI TOF) mass spectrometer (bioMérieux, Inc., Durham, NC) as Candida boidinii. API 20C System (bioMérieux, Inc., Durham, NC) identification yielded profiles that were nondescriptive. A definitive identification of C. fabianii for both morphotypes was achieved through sequencing of the D1/D2 region of the large subunit of $28 \mathrm{~S}$ ribosomal RNA gene and internal transcribed spacer (ITS) region [18]. Antifungal susceptibility testing was performed according to CLSI standards by broth microdilution in RPMI, in panels prepared in-house [19]. Antifungal minimum inhibitory concentrations (MIC) for fluconazole, flucytosine,

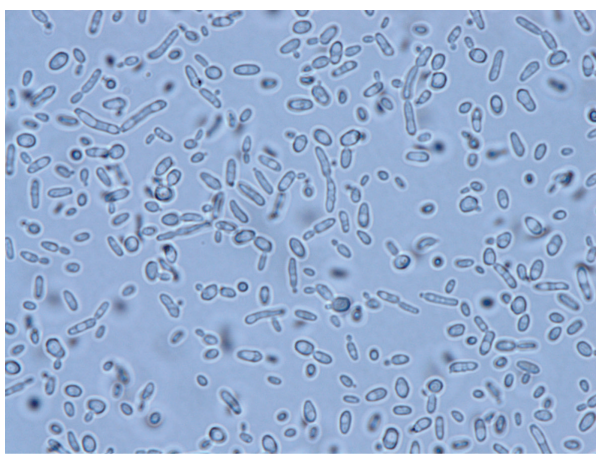

FIGURE 1: Wet mount preparation of C. fabianii from growth on IMA, demonstrating budding yeast cells and elongated pseudohyphae.

and amphotericin B were $2.0,<0.12$, and $2.0 \mathrm{mg} / \mathrm{L}$, respectively.

Follow-up detailed history for possible environmental exposures revealed that the patient had ingested multiple servings of cơm rượu, a traditional Vietnamese dessert prepared by fermenting rice, 5-7 days before the onset of illness. The patient denied ingestion of raw milk and cheese.

The patient was discharged home after 11 days, still receiving intravenous liposomal amphotericin $\mathrm{B}$ and oral flucytosine. After 19 days of antifungals, she developed acute kidney injury and her regimen was changed to monotherapy with oral fluconazole. She developed painful cheilitis with fluconazole $800 \mathrm{mg}$ daily and thus had her dose adjusted to $400 \mathrm{mg}$. At 6-month follow-up, she was feeling better and had returned to baseline function. She will continue fluconazole indefinitely to prevent recrudescence of her infection in the setting of retained VP shunt components.

\section{Discussion}

Fungal meningitis is an uncommon but serious condition affecting neurosurgical patients with VP shunts $[1,4]$. Patients tend to present with fever and symptoms of shunt malfunction, such as headache, nausea, and altered mental status $[1,4]$. In particular, Candida spp. have been identified as the cause of $\sim 1 \%$ of VP shunt infections [1-4]. Up to $77 \%$ of Candida VP shunt infections develop within three months of shunt manipulation, suggesting inoculation of the organism during surgery [1-4]. Other risk factors include recent abdominal surgery, bowel perforation, use of broad-spectrum antibiotics, steroids, vascular catheters, prior or concurrent meningitis, and CSF leak [1,4]. Transient fungemia in the absence of the above risk factors has been suggested as a mechanism that may lead to VP shunt fungal infection [5], but evidence is lacking. The current case demonstrates that transient fungemia with a yeast that is not part of the normal gastrointestinal flora may has occurred in the setting of ingestion of multiple servings of a fermentation product despite lack of known bowel disease. Thus, this case provides indirect evidence that supports the hypothesis that transient 
fungemia in immunocompetent patients without any other risk factors for fungemia may lead to serious fungal VP shunt infections, even with a remote history of VP placement.

Candida fabianii is an environmental yeast with similarities to Candida spp. Members of the genus Cyberlindnera exhibit phenotypic traits shared by members of the genera Pichia, Issatchenkia, and Williopsis, including production of ubiquinone CoQ-7 and inability to utilize methanol [7]. As with other heterothallic ascomycetous yeasts, C. fabianii is capable of sexual reproduction in which an ascus is formed containing small hat-shaped ascospores [20]. Despite being not typically performed in a clinical laboratory, organisms in this group can be induced to form ascospores using special sporulation media such as V8 agar and Gorodkowa agar [21]. Identification of this particular yeast species can be problematic [16]. Commercial identification platforms such as Vitek 2 with ID 32C system have been shown to incorrectly identify C. fabianii as Candida pelliculosa or Candida utilis [10]. Although MALDI-TOF has been shown in a recent report to be able to identify clinically relevant Candida spp., it failed to identify C. fabianii, whose spectral profile was not included in the Saramis reference database used with the Vitek MS [22]. Antifungal susceptibility testing may be performed on this organism using broth dilution method as for other yeasts, though there are currently no interpretive criteria [23].

Infections due to C. fabianii are extremely rare and have to date not involved the central nervous system. Details of the 14 reported cases are summarized in Table 1 . Cases have been described in 9 neonates and 5 adults (average age of 48.6, range of 40-57). Three cases developed in individuals who were not known to be immunocompromised $[9,10,14]$. Three patients suffered from concurrent colitis $[9,11,14]$. Nine of ten (90\%) fungemias developed in the setting of broad-spectrum antibiotics [9-16]. In all cases, C. fabianii was isolated by culture. In 13 of 14 cases (92.9\%), species identification was confirmed by molecular sequencing. Antifungal susceptibility varies, with 10 of $14(71.4 \%)$ cases involving isolates with MICs $\leq 2 \mu \mathrm{g} / \mathrm{mL}$ for fluconazole [8-11]. Mlinarić-Missoni et al. recently described the susceptibility profiles of the 44 isolates (from six patients) of C. fabianii to amphotericin $\mathrm{B}$, flucytosine, triazoles, and echinocandins and found a higher proportion of nonsusceptible isolates of C. fabianii for micafungin compared with the other tested antifungals [16]. No in vitro resistance to amphotericin B, flucytosine, fluconazole, itraconazole, or voriconazole was observed [16].

Treatments have been various, with amphotericin $\mathrm{B}$, fluconazole, and caspofungin all used successfully. In two cases (14.3\%), major surgery was required for source control $[10,13]$. Microbiological and clinical cure was achieved in 11 cases $(78.6 \%)[8,10,11,13,14,16]$. In the remaining three cases, the infected patient did not survive $[9,12,15]$.

Pichia species are often found in raw milk, cheese, and traditional fermented products and a case of Pichia anomala (Candida pelliculosa) fungemia from ingestion of raw milk and yogurt has been published [24]. Candida fabianii has been isolated from fermentation products [25] and homemade alcoholic beverages [26]. We suspect our patient acquired C. fabianii via ingestion of cơm rượu, a traditional
Vietnamese dessert made from the fermentation of rice. We hypothesize that $C$. fabianii may have inoculated the peritoneal terminus of her VP shunt via translocation from the gut, either directly across the peritoneal space or by transient fungemia. This hypothesis is supported by the predilection of C. fabianii for neonates-whose gut mucosal immunity is undeveloped-and adults with colitis. A similar mechanism of infection has been proposed for Saccharomyces cerevisiae, which has caused fungemia in immunocompetent individuals ingesting the organism as a probiotic [27]. This case suggests for the first time that C. fabianii may lead to invasive disease in an immunocompetent host with a remote history of VP shunt placement after ingestion of commercially available fermentation products.

Given the rarity of C. fabianii infections, no guidelines are available to direct treatment. Decisions regarding antifungal therapy must be individualized. Amphotericin B and flucytosine were chosen initially, prior to determination of the species of the yeast and its antifungal susceptibility, based on clinical guidelines for central nervous system candidiasis [28]. By the time C. fabianii was identified, the patient had already recovered symptomatically, achieved microbiologic cure, and been transitioned to oral fluconazole for maintenance. Clinical studies of adults with cryptococcal meningitis have shown that a dose of fluconazole $400 \mathrm{mg}$ daily achieves average CSF concentrations of $25.1 \mathrm{mg} / \mathrm{L}$, a concentration at least 10 times higher than the MICs of our two isolates [29]. Further, there is a precedent for the use of fluconazole $400 \mathrm{mg}$ daily for maintenance therapy in the management of cryptococcal meningitis [30]. In other instances of fungal meningitis, removal of indwelling devices such as VP shunts is considered standard of care [31,32]. Unfortunately, components of our patient's infected VP shunt remain in place. Thus, she will likely require lifelong antifungal therapy. If recurrent infection develops, repeat susceptibility testing will need to be performed, as at least one case of $C$. fabianii has been reported to develop an elevated MIC to fluconazole and voriconazole during prolonged therapy [10]. In a case series of 6 cases of $C$. fabianii infection, fluconazole administration either prophylactically or therapeutically did not lead to resolution of the infection in $50 \%$ of the patients [16]. Thus, every effort should be made for complete removal of VP shunts infected with $C$. fabianii since in certain cases long-term oral suppression therapy with an azole may not be an option.

\section{Conclusions}

Clinicians should be aware of the clinical presentation, diagnostic methods, and therapeutic options for treatment of $C$. fabianii infection, as this organism may cause life-threatening infections including CNS infections. Transient fungemia may lead to invasive disease in an immunocompetent host with VP shunt, even in the absence of any other risk factors for fungemia and even after remote placement of the VP shunt. In view of the high morbidity associated with yeast infection of the CNS, long-term antifungal therapy should be strongly considered in cases where the VP shunt cannot be completely removed. 


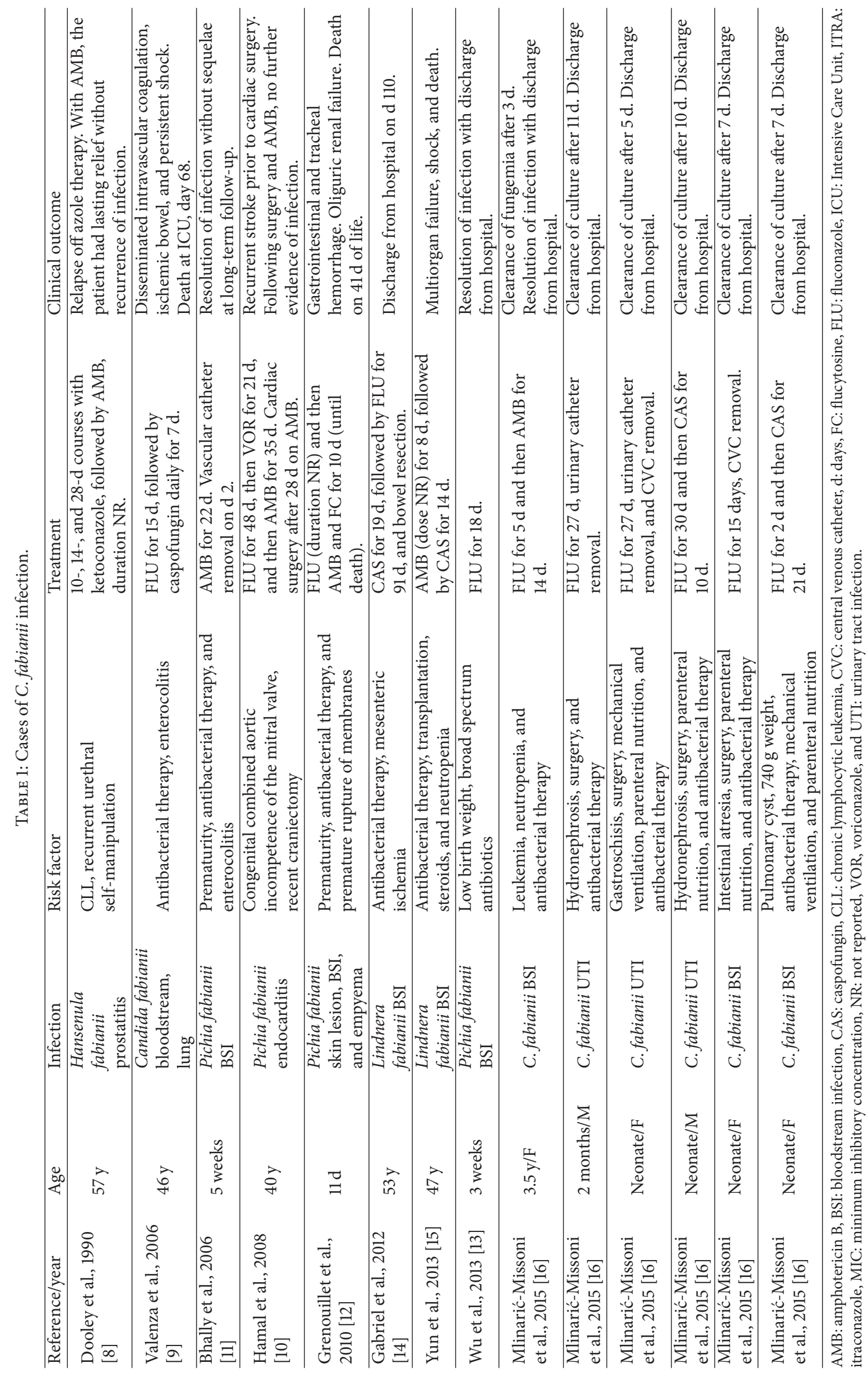




\section{Conflict of Interests}

The authors declare that there is no conflict of interests regarding the publication of this paper.

\section{References}

[1] E. M. Kojic and R. O. Darouiche, "Candida infections of medical devices," Clinical Microbiology Reviews, vol. 17, no. 2, pp. 255267, 2004

[2] V. P. Baradkar, M. Mathur, A. Sonavane, and S. Kumar, "Candidal infections of ventriculoperitoneal shunts," Journal of Pediatric Neurosciences, vol. 4, no. 2, pp. 73-75, 2009.

[3] J. Sánchez-Portocarrero, P. Martín-Rabadán, C. J. Saldaña, and E. Pérez-Cecilia, "Candida cerebrospinal fluid shunt infection. Report of two new cases and review of the literature," Diagnostic Microbiology and Infectious Disease, vol. 20, no. 1, pp. 33-40, 1994.

[4] M. H. Nguyen and V. L. Yu, "Meningitis caused by Candida species: an emerging problem in neurosurgical patients," Clinical Infectious Diseases, vol. 21, no. 2, pp. 323-327, 1995.

[5] S. Shapiro, T. Javed, and J. Mealey Jr., "Candida albicans shunt infection,” Pediatric Neuroscience, vol. 15, no. 3, pp. 125-130, 1989.

[6] K. C. Freel, V. Sarilar, C. Neuveglise, H. Devillers, A. Friedrich, and J. Schacherer, "Genome sequence of the yeast Cyberlindnera fabianii (Hansenula fabianii)," Genome Announcements, vol. 2, no. 4, 2014.

[7] C. P. Kurtzman, C. J. Robnett, and E. Basehoar-Powers, "Phylogenetic relationships among species of Pichia, Issatchenkia and Williopsis determined from multigene sequence analysis, and the proposal of Barnettozyma gen. nov., Lindnera gen. nov. and Wickerhamomyces gen. nov," FEMS Yeast Research, vol. 8, no. 6, pp. 939-954, 2008.

[8] D. P. Dooley, M. L. Beckius, C. K. McAllister, and B. S. Jeffery, "Prostatitis caused by Hansenula fabianii," Journal of Infectious Diseases, vol. 161, no. 5, pp. 1040-1041, 1990.

[9] G. Valenza, R. Valenza, J. Brederlau, M. Frosch, and O. Kurzai, "Identification of Candida fabianii as a cause of lethal septicaemia," Mycoses, vol. 49, no. 4, pp. 331-334, 2006.

[10] P. Hamal, J. Ostransky, M. Dendis et al., "A case of endocarditis caused by the yeast Pichia fabianii with biofilm production and developed in vitro resistance to azoles in the course of antifungal treatment," Medical Mycology, vol. 46, no. 6, pp. 601-605, 2008.

[11] H. S. Bhally, S. Jain, C. Shields, N. Halsey, E. Cristofalo, and W. G. Merz, "Infection in a neonate caused by Pichia fabianii: importance of molecular identification," Medical Mycology, vol. 44, no. 2, pp. 185-187, 2006.

[12] F. Grenouillet, L. Millon, A. Chamouine, G. Thiriez, O. Schulze, and J. Leroy, "Pichia fabianii fungemia in a neonate," Pediatric Infectious Disease Journal, vol. 29, no. 2, p. 191, 2010.

[13] Y. Wu, J. Wang, W. Li et al., "Pichia fabianii blood infection in a premature infant in China: case report," BMC Research Notes, vol. 6, article 77, 2013.

[14] F. Gabriel, T. Noel, and I. Accoceberry, "Lindnera (Pichia) fabianii blood infection after mesenteric ischemia," Medical Mycology, vol. 50, no. 3, pp. 310-314, 2012.

[15] J. W. Yun, K. S. Park, C.-S. Ki, and N. Y. Lee, "Catheter-related bloodstream infection by Lindnera fabianii in a neutropenic patient," Journal of Medical Microbiology, vol. 62, no. 6, pp. 922925, 2013.
[16] E. H. Mlinarić-Missoni, L. Hatvani, S. Kocsubé, C. Vágvölgyi, I. Škarić, and A. Lukić-Grlić, "Cyberlindnera fabianii in the neonatal and paediatric intensive care unit: case reports," JMM Case Reports, vol. 2, no. 3, 2015.

[17] T. Scognamiglio, R. Zinchuk, P. Gumpeni, and D. H. Larone, "Comparison of inhibitory mold agar to sabouraud dextrose agar as a primary medium for isolation of fungi," Journal of Clinical Microbiology, vol. 48, no. 5, pp. 1924-1925, 2010.

[18] S. N. Leaw, H. C. Chang, H. F. Sun, R. Barton, J.-P. Bouchara, and T. C. Chang, "Identification of medically important yeast species by sequence analysis of the internal transcribed spacer regions," Journal of Clinical Microbiology, vol. 44, no. 3, pp. 693699, 2006.

[19] Clinical and Laboratory Standards Institute (CLSI), Reference Method for Broth Dilution Antifungal Susceptibility Testing of Yeasts; Fourth Informational Supplement (CLSI Document M27S4), Clinical and Laboratory Standards Institute, Wayne, Pa, USA, 2012.

[20] L. J. Wickerham, "New Heterothallic species of Hansenula," Mycopathologia et Mycologia Applicata, vol. 26, no. 1, pp. 79-86, 1965.

[21] A. E. Merritt and R. Hurley, "Evaluation of sporulation media for yeasts obtained from pathological material," Journal of Medical Microbiology, vol. 5, no. 1, pp. 21-30, 1972.

[22] R. L. Gorton, S. Seaton, P. Ramnarain, T. D. McHugh, and C. C. Kibbler, "Evaluation of a short, on-plate formic acid extraction method for matrix-assisted laser desorption ionization-time of flight mass spectrometry-based identification of clinically relevant yeast isolates," Journal of Clinical Microbiology, vol. 52, no. 4, pp. 1253-1255, 2014.

[23] Clinical and Laboratory Standards Institute (CLSI), Performance Standards for Antimicrobial Disk and Dilution Susceptibility Tests for Bacteria Isolated From Animals; Second Informational Supplement (VET01-S2), Clinical and Laboratory Standards Institute, Wayne, Pa, USA, 2013.

[24] A. W. Chan, E. J. Cartwright, S. C. Reddy, C. S. Kraft, and Y. F. Wang, "Pichia anomala (Candida pelliculosa) fungemia in a patient with sickle cell disease," Mycopathologia, vol. 176, no. 34, pp. 273-277, 2013.

[25] L. K. Nyanga, M. J. R. Nout, E. J. Smid, T. Boekhout, and M. H. Zwietering, "Fermentation characteristics of yeasts isolated from traditionally fermented masau (Ziziphus mauritiana) fruits," International Journal of Food Microbiology, vol. 166, no. 3, pp. 426-432, 2013.

[26] K. Jeyaram, W. M. Singh, A. Capece, and P. Romano, "Molecular identification of yeast species associated with 'Hamei' - a traditional starter used for rice wine production in Manipur, India," International Journal of Food Microbiology, vol. 124, no. 2, pp. 115-125, 2008.

[27] P. Muñoz, E. Bouza, M. Cuenca-Estrella et al., "Saccharomyces cerevisiae fungemia: an emerging infectious disease," Clinical Infectious Diseases, vol. 40, no. 11, pp. 1625-1634, 2005.

[28] P. G. Pappas, C. A. Kauffman, D. Andes et al., "Clinical practice guidelines for the management of candidiasis: 2009 update by the infectious diseases Society of America," Clinical Infectious Diseases, vol. 48, no. 5, pp. 503-535, 2009.

[29] W. Manosuthi, P. Chetchotisakd, T. L. Nolen et al., "Monitoring and impact of fluconazole serum and cerebrospinal fluid concentration in HIV-associated cryptococcal meningitis-infected patients," HIV Medicine, vol. 11, no. 4, pp. 276-281, 2010.

[30] L. M. Mofenson, M. T. Brady, S. P. Danner et al., "Guidelines for the prevention and treatment of opportunistic infections 
among HIV-exposed and HIV-infected children: recommendations from CDC, the National Institutes of Health, the HIV Medicine Association of the Infectious Diseases Society of America, the Pediatric Infectious Diseases Society, and the American Academy of Pediatrics," MMWR Recommendations and Reports, vol. 58, no. RR11, pp. 1-166, 2009.

[31] D. O'Brien, N. T. Stevens, C. H. Lim et al., "Candida infection of the central nervous system following neurosurgery: a 12-year review," Acta Neurochirurgica, vol. 153, no. 6, pp. 1347-1350, 2011.

[32] M. J. Viereck, N. Chalouhi, D. I. Krieger, and K. D. Judy, "Cryptococcal ventriculoperitoneal shunt infection," Journal of Clinical Neuroscience, vol. 21, no. 11, pp. 2020-2021, 2014. 


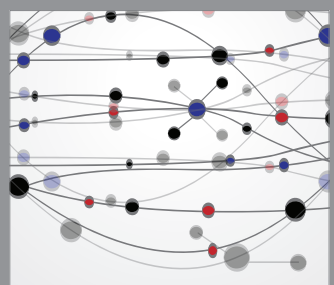

The Scientific World Journal
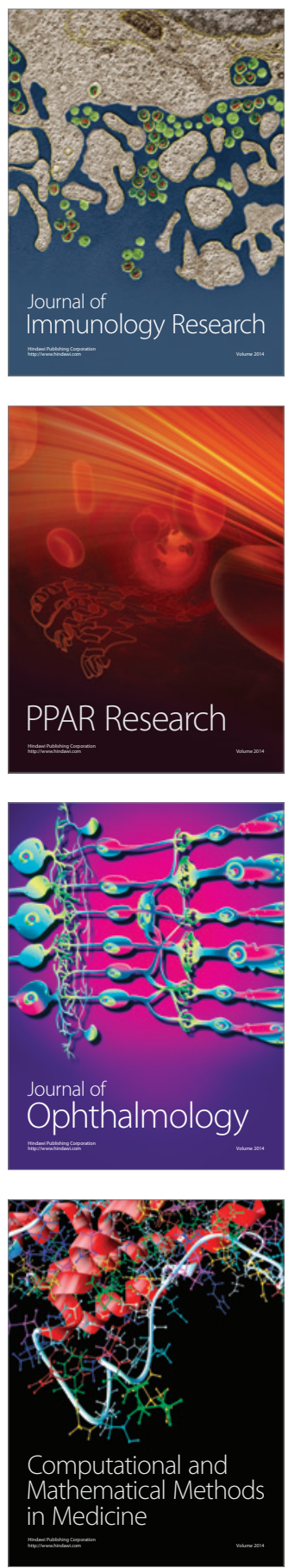

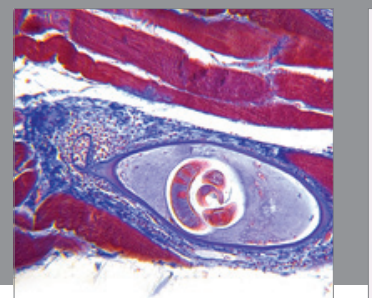

Gastroenterology

Research and Practice
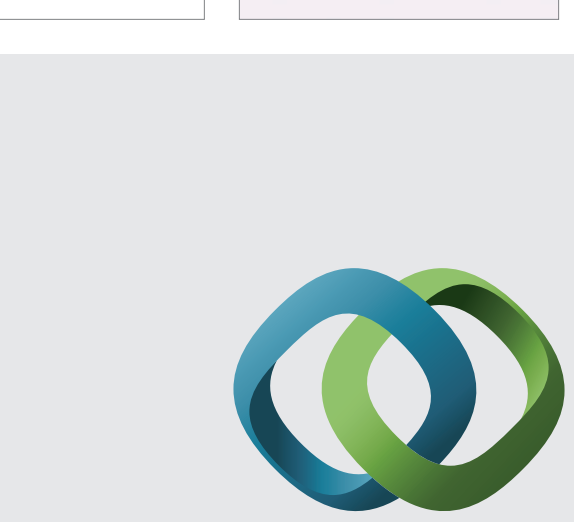

\section{Hindawi}

Submit your manuscripts at

http://www.hindawi.com
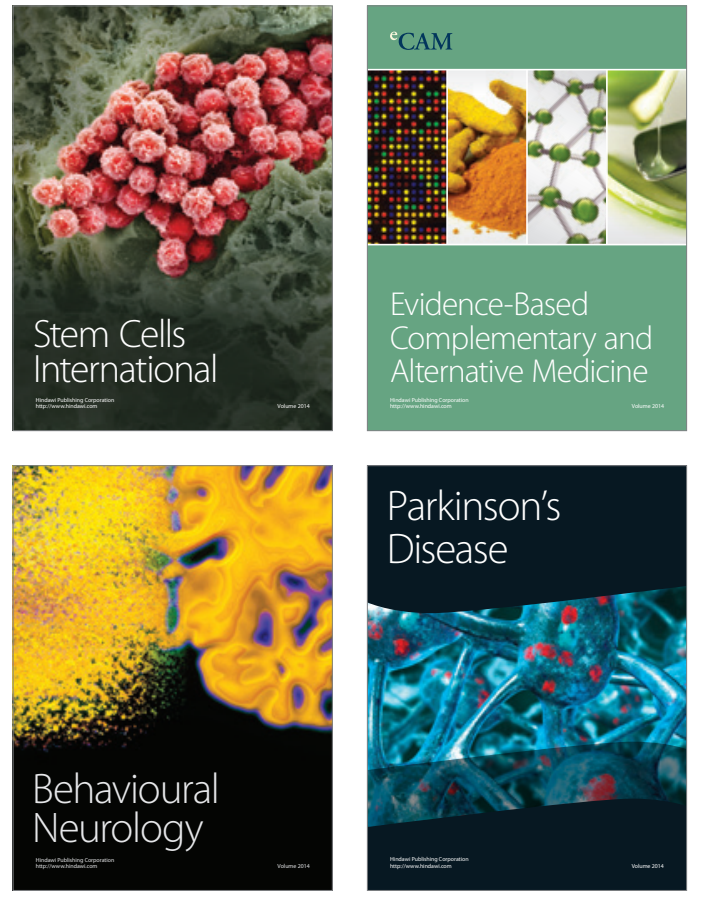
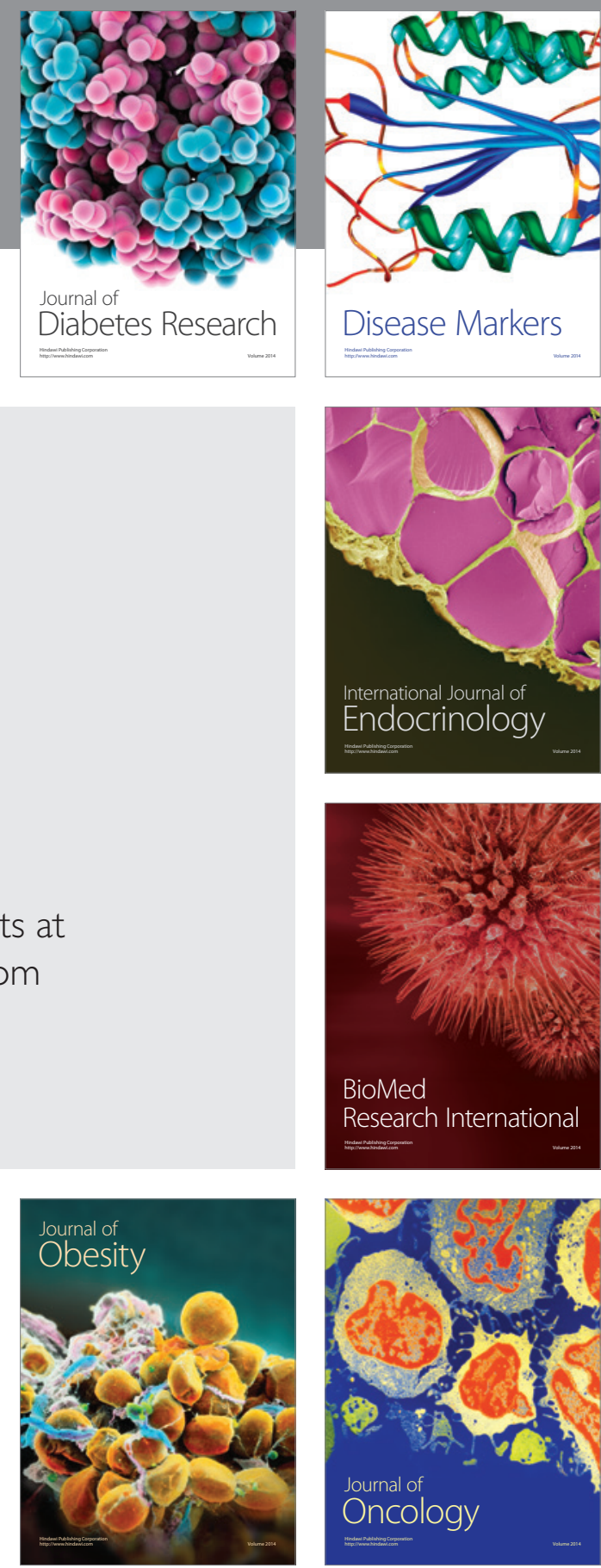

Disease Markers
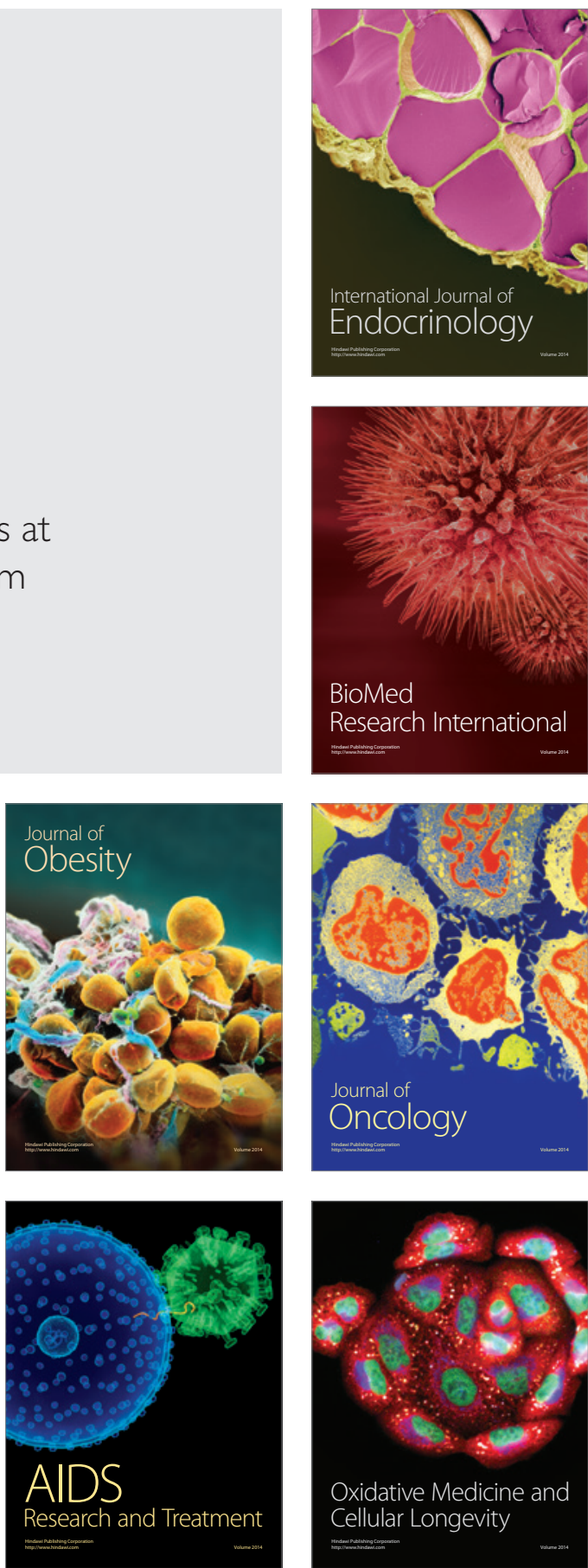